\title{
Early Age-Related Functional Connectivity Decline in High-Order Cognitive Networks
}

\author{
Tali Siman-Tov' ${ }^{*}$, Noam Bosak ${ }^{2}$, Elliot Sprecher ${ }^{3,4}$, Rotem Paz ${ }^{1}$, Ayelet Eran ${ }^{5}$, \\ Judith Aharon-Peretz ${ }^{1,4}$ and Itamar Kahn ${ }^{2 *}$
}

${ }^{1}$ Cognitive Neurology Institute, Rambam Health Care Campus, Haifa, Israel, ${ }^{2}$ Department of Neuroscience, Ruth and Bruce Rappaport Faculty of Medicine, Technion - Israel Institute of Technology, Haifa, Israel, ${ }^{3}$ Laboratory of Clinical Neurophysiology, Ruth and Bruce Rappaport Faculty of Medicine, Technion - Israel Institute of Technology, Haifa, Israel, ${ }^{4}$ Department of Neurology, Rambam Health Care Campus, Haifa, Israel, ${ }^{5}$ Department of Diagnostic Imaging, Rambam Health Care Campus, Haifa, Israel

\section{OPEN ACCESS \\ Edited by: \\ Ashok Kumar, \\ University of Florida, USA}

Reviewed by:

R. Nathan Spreng,

Cornell University, USA

Natalie Ebner,

University of Florida, USA

*Correspondence:

Tali Siman-Tov

simantov.tali@gmail.com

Itamar Kahn

kahn@technion.ac.il

Received: 21 August 2016 Accepted: 19 December 2016 Published: 10 January 2017

Citation:

Siman-Tov T, Bosak N, Sprecher E,

Paz R, Eran A, Aharon-Peretz $J$ and Kahn I (2017) Early Age-Related Functional Connectivity Decline

in High-Order Cognitive Networks.

Front. Aging Neurosci. 8:330.

doi: 10.3389/fnagi.2016.00330
As the world ages, it becomes urgent to unravel the mechanisms underlying brain aging and find ways of intervening with them. While for decades cognitive aging has been related to localized brain changes, growing attention is now being paid to alterations in distributed brain networks. Functional connectivity magnetic resonance imaging (fcMRI) has become a particularly useful tool to explore large-scale brain networks; yet, the temporal course of connectivity lifetime changes has not been established. Here, an extensive cross-sectional sample (21-85 years old, $N=887$ ) from a public fcMRl database was used to characterize adult lifespan connectivity dynamics within and between seven brain networks: the default mode, salience, dorsal attention, frontoparietal control, auditory, visual and motor networks. The entire cohort was divided into young (21-40 years, mean \pm SD: $25.5 \pm 4.8, n=543)$; middle-aged (4160 years, $50.6 \pm 5.4, n=238$ ); and old (61 years and above, $69.0 \pm 6.3, n=106$ ) subgroups. Correlation matrices as well as a mixed model analysis of covariance indicated that within high-order cognitive networks a considerable connectivity decline is already evident by middle adulthood. In contrast, a motor network shows increased connectivity in middle adulthood and a subsequent decline. Additionally, alterations in inter-network interactions are noticeable primarily in the transition between young and middle adulthood. These results provide evidence that aging-related neural changes start early in adult life.

Keywords: aging, brain networks, functional connectivity, lifespan, MRI, resting state

\section{INTRODUCTION}

Aging has long been suggested to be accompanied by cognitive decline, even in the absence of dementia or other neurological insults. This decline is more pronounced in cognitive abilities such as processing speed, working memory, and encoding new information into episodic memory, whereas other capacities, particularly semantic knowledge, and emotional processing, seem to remain relatively stable along the adult lifespan (Park et al., 2002; Buckner, 2004; Hedden and Gabrieli, 2004; Park and Payer, 2006; Salthouse, 2009). The precise determination of agerelated cognitive changes remains a challenge due to high variability across cognitive skills and research designs. Largely, a monotonous, linear to a first approximation, decline has been reported, starting in the third decade (Buckner, 2004; Park and Reuter-Lorenz, 2009), with some 
cognitive abilities exhibiting an accelerated deterioration in the seventh decade (Hedden and Gabrieli, 2004).

Age-associated cognitive impairment has frequently been linked to structural modifications in both gray matter (GM) and white matter (WM) (Buckner, 2004; Madden et al., 2009; Bergfield et al., 2010; Giorgio et al., 2010; Thambisetty et al., 2010), though temporal characteristics of these changes and the exact relations with cognitive performance have not been defined (Ferreira et al., 2014). GM volume loss has typically been reported as linear, beginning early in life (Good et al., 2001; Ge et al., 2002; Giorgio et al., 2010; Chen et al., 2013) and only few studies indicated non-linear trends (Terribilli et al., 2011; Lebel et al., 2012; Ziegler et al., 2012). Conversely, WM volume dynamics has frequently been described as non-linear with an inverted$\mathrm{U}$ trajectory over the entire lifespan peaking in the fourth to fifth decade (Giorgio et al., 2010; Chen et al., 2013). Further, fractional anisotropy (FA), a diffusion tensor imaging (DTI) index thought to reflect WM integrity, was shown to deteriorate earlier than WM volume (Salat et al., 2005; Giorgio et al., 2010), quickly after peaking between 20 and 42 years of age (Lebel et al., 2012). Interestingly, a few DTI studies reported a considerable FA decrement in the transition between early and middle adulthood, whereas no significant difference was observed between middleaged and older adults (Salat et al., 2005; Giorgio et al., 2010).

In addition to structural modifications, alterations in neurotransmitter function have been described in healthy aging, particularly reductions in the binding potential of monoaminergic transmitters (Hedden and Gabrieli, 2004; Park and Reuter-Lorenz, 2009; Bäckman et al., 2010). Age-related cognitive deficits have been most associated with dopaminergic dysfunction (Volkow et al., 1998; Bäckman et al., 2000, 2006; Erixon-Lindroth et al., 2005), though the exact trajectory of dopaminergic decline is still disputed with both linear and non-linear reductions documented (Bäckman et al., 2006). Of note are a few reports highlighting a dramatic decline by midlife in the availability of dopamine receptors (Antonini et al., 1993), dopamine transporters (Mozley et al., 1996), and serotonin receptors (Sheline et al., 2002); yet, these are awaiting confirmation by larger and wider age-range studies.

Importantly, several other neurobiological measures potentially related to cognitive aging are greatly modified by middle-age. For instance, deficits in adult dentate and subventricular zone neurogenesis, considered to play a role in aging, were reported as already established in middle-aged animals (Kuhn et al., 1996; Hamilton et al., 2013). The levels of brain-derived neurotrophic factor (BDNF), phosphorylated cAMP response element binding protein (p-CREB) and neuropeptide Y (NPY), recognized as positive regulators of dentate neurogenesis and memory function, significantly declined as early as middle-age in the rat hippocampus (Hattiangady et al., 2005). Moreover, age-related synaptic plasticity changes, such as long-term potentiation deficits, were evident in middle-aged animals (Rex et al., 2005; Lynch et al., 2006). In humans, signal pathway activation (e.g., ERK-, JNK-, mTOR-, MAPK-, mitochondrial apoptosis, and caspase cascade) in fibroblasts derived from young, middle-aged and old healthy adults as well as Progeria patients showed more pronounced alterations in the transition between the young and middleaged groups than among other groups (Aliper et al., 2015). Further, a set of genes related particularly to synaptic plasticity, vesicular transport and mitochondrial function showed reduced expression in the frontal cortex after age 40 (Lu et al., 2004). Collectively, this evidence from animal models and humans implies that some age-related decline may present in middle-age.

The timing of age-related structural modifications was reported to vary across brain regions with earlier deterioration in high-order association areas relative to primary sensory and motor cortices (Raz, 2000). Association regions are known to develop relatively late, both ontogenetically and phylogenetically, compared to primary sensory and motor areas (Tau and Peterson, 2010; Fjell et al., 2014); therefore age-related brain changes were suggested to follow the "last-in-first-out" rule (Raz, 2001). Today, brain aging is thought to involve not only regional dysfunction but also alterations in the communication between remote brain areas (Andrews-Hanna et al., 2007; Antonenko and Floel, 2014). In addition to WM structural connections evaluated by means of, for example, DTI, functional connections between distinct GM regions are also explored. Analysis of coherent spontaneous fluctuations in the fMRI blood oxygenation level-dependent (BOLD) signal, termed intrinsic functional connectivity MRI (fcMRI; for reviews, see Fox and Raichle, 2007; Buckner et al., 2013) now allows studying the brain's connectional architecture across the lifespan. Heretofore, several fcMRI studies have documented age-related reduced connectivity within large-scale intrinsic connectivity networks (ICNs) (Andrews-Hanna et al., 2007; Damoiseaux et al., 2008; Koch et al., 2010; Tomasi and Volkow, 2012; Ferreira and Busatto, 2013), and altered connectivity between ICNs has also been proposed (Onoda et al., 2012; Antonenko and Floel, 2014). However, this literature refers to only a few ICNs, lifespan trajectories of intranetwork connectivity decline and differences between networks are not well characterized and results regarding inter-network connectivity changes are variable, with both increases (Meunier et al., 2009; Betzel et al., 2014; Chan et al., 2014; Geerligs et al., 2015; Grady et al., 2016; Spreng et al., 2016) and decreases (Meunier et al., 2009; Allen et al., 2011; Onoda et al., 2012) reported.

The present study aims to fill this gap and extend our view of intra- and inter-network functional reorganization during aging. The authors believe that a comprehensive outlook of the lifetime dynamics of brain functional connectomics is crucial for deciphering the mechanisms underlying brain aging, determining intervention targets and their optimal timing. Herein, an extensive fcMRI cohort of 887 healthy individuals between the ages of 21 and 85 was used to characterize functional connectivity changes across the adult lifespan in seven robust well-established ICNs: the default mode (DMN), salience (SN), dorsal attention (DAN), fronto-parietal control (FPCN), auditory $(\mathrm{AN})$, visual $(\mathrm{VN})$, and motor $(\mathrm{MN})$ networks (Damoiseaux et al., 2006; Van den Heuvel and Hulshoff Pol, 2010; Van Dijk et al., 2010; Yeo et al., 2011; Brier et al., 2012). Consistent with previous lifespan studies (Giorgio et al., 2010; Van Helmond et al., 2010; Park et al., 2013; Ferreira et al., 2015; Kennedy et al., 2015), the cohort was divided into young, middle-aged, 
and old groups. Consistent with earlier imaging literature ( $\mathrm{Li}$ et al., 2011, 2013; Montembeault et al., 2012; Belcher et al., 2013; Geerligs et al., 2015) the seven networks were divided into highorder cognitive networks (DMN, SN, DAN, and FPCN), known to involve associative brain areas and play a role in high-order cognition (Van den Heuvel et al., 2009; Menon, 2011; Braga and Leech, 2015) and primary sensory and motor networks (AN, $\mathrm{VN}$, and MN). Differential response of these network groups to aging was assumed based on recent fcMRI reports (Chan et al., 2014; Geerligs et al., 2015) and the "last-in-first-out" theory (Raz, 2001). Taken together, we expected functional connectivity decline by middle-age within high-order cognitive networks, as well as early between-network connectivity alterations, where high-order cognitive networks are involved.

\section{MATERIALS AND METHODS}

\section{Participants}

Intrinsic functional connectivity MRI data recorded at rest of 887 healthy individuals (age range 21-85 years, 514 females) were obtained from the online dataset of the International Neuroimaging Data-sharing Initiative (INDI), '1000 Functional Connectomes' Project ${ }^{1}$ (Mennes et al., 2013). The entire cohort was subdivided into three groups: young (21-40 years, mean \pm SD: $25.5 \pm 4.8, n=543,284$ females); middleaged (41-60 years, $50.6 \pm 5.4, n=238,160$ females); and old (61 years and above, $69.0 \pm 6.3, n=106,70$ females). Seventeen research sites were included (Table 1; Supplementary Figure S1). Each center's ethics committee approved submission of de-identified data. The institutional review board of Rambam healthcare campus approved the receipt and analysis of these data. Centers including less than 10 participants and data of participants with partial brain coverage were discarded from analysis. Sixteen additional individuals were not included due to excessive head movement. Three hundred and eighteen out of the 887 participants (Table 2) were selected for further statistical analysis as detailed below.

\section{Functional Imaging Data Acquisition and Preprocessing}

Acquisition data are shown in Table 1. Functional images were preprocessed using FSL software (FMRIB Software Library v. 5.0.1, Oxford, UK) and SPM software (Statistical Parametric Mapping software package, Wellcome Department of Imaging Neuroscience, London, UK) following conventional methods as previously described (Kahn et al., 2008; Kahn and Shohamy, 2013). Preprocessing included rigid body correction for motion within and across runs (FSL), normalization to the standard EPI template of the Montreal Neurological Institute (MNI) and compensation for slice-dependent time shifts (SPM). The preprocessed functional data (in atlas space) were then temporally filtered to remove constant offsets and linear trends over each run while retaining frequencies below $0.08 \mathrm{~Hz}$. Data were spatially smoothed using a $4 \mathrm{~mm}$ full-width half-maximum

\footnotetext{
${ }^{1}$ http://fcon_1000.projects.nitrc.org/
}

Gaussian blur. Sources of spurious or regionally non-specific variance were removed by regression of nuisance variables including six parameters obtained by rigid body head motion correction, the signal averaged over the whole brain (global signal), the signal averaged over the lateral ventricles and the signal averaged over a region centered in the deep cerebral WM. Temporally shifted versions of these waveforms were removed by inclusion of the first temporal derivatives (computed by backward differences) in the linear model. The data was also analyzed without global signal regression (GSR) to evaluate its potential influence on our findings.

\section{Functional Connectivity Analysis}

Seed-based analysis was performed as previously described (Fox et al., 2005; Kahn et al., 2008; Van Dijk et al., 2010) to study four high-order cognitive networks: DMN, SN, DAN, and FPCN as well as three primary sensory and motor networks: AN, VN, and MN. To delineate these networks, the following seed regions were used: left posterior cingulate cortex (LPCC); right frontoinsula (RFI); right intraparietal sulcus (RIPS); right superior parietal cortex (RSP); left auditory cortex (LAC); right visual cortex (RVC); and left motor cortex (LMC). Each seed was defined as a $6 \mathrm{~mm}$ radius sphere centered on previously published foci (Table 3). Correlation maps were produced by extracting the time course from each of the above seeds and computing the Pearson correlation coefficient $(r)$ between this time course and the time course of each voxel across the whole brain. SPM8 software was used to compute statistical maps of each network across participants. Maps of a young age group (458 participants, 21-30 years) were used to identify peak coordinates of additional regions of interest (ROIs) representing each network. All regions were defined as $6 \mathrm{~mm}$ radius spheres around the peak coordinate (Table 3). For statistical tests, the Fischer's $r$-to- $z$ transformation $(z(r)=0.5 \ln [(1+r) /(1-r)])$ was applied. Using MATLAB version R2012b (Mathworks, Natick, MA, USA), node pair correlation matrices were computed for the three age groups: young (21-40 years), middle-aged (4160 years), and old (61 years and above). Additionally, between age group difference matrices were calculated for the young vs. the middle-aged group and the middle-aged vs. the old group. These matrices exhibit the age group difference in connectivity strength $[z(r)$ value $]$ for each node pair. Correction for multiple comparisons was carried out using the false discovery rate (FDR) procedure (Benjamini and Yekutieli, 2001) implemented in MATLAB with no assumptions about test dependency and at an FDR level of 0.05. Group difference maps for the DMN and MN were computed using the two-sample $t$-test implemented in SPM8. Three significant ROIs were chosen for each of the four contrasts to present $z(r)$ values of ROI-tonetwork seed (LPCC or LMC) across participants within each age group. Regions were defined as $4 \mathrm{~mm}$ radius spheres around the peak coordinate. Box-and-whisker plots were drawn using MATLAB.

\section{Head Motion Correction}

As head motion is widely considered a significant confound in fcMRI (Power et al., 2012; Van Dijk et al., 2012) and in 


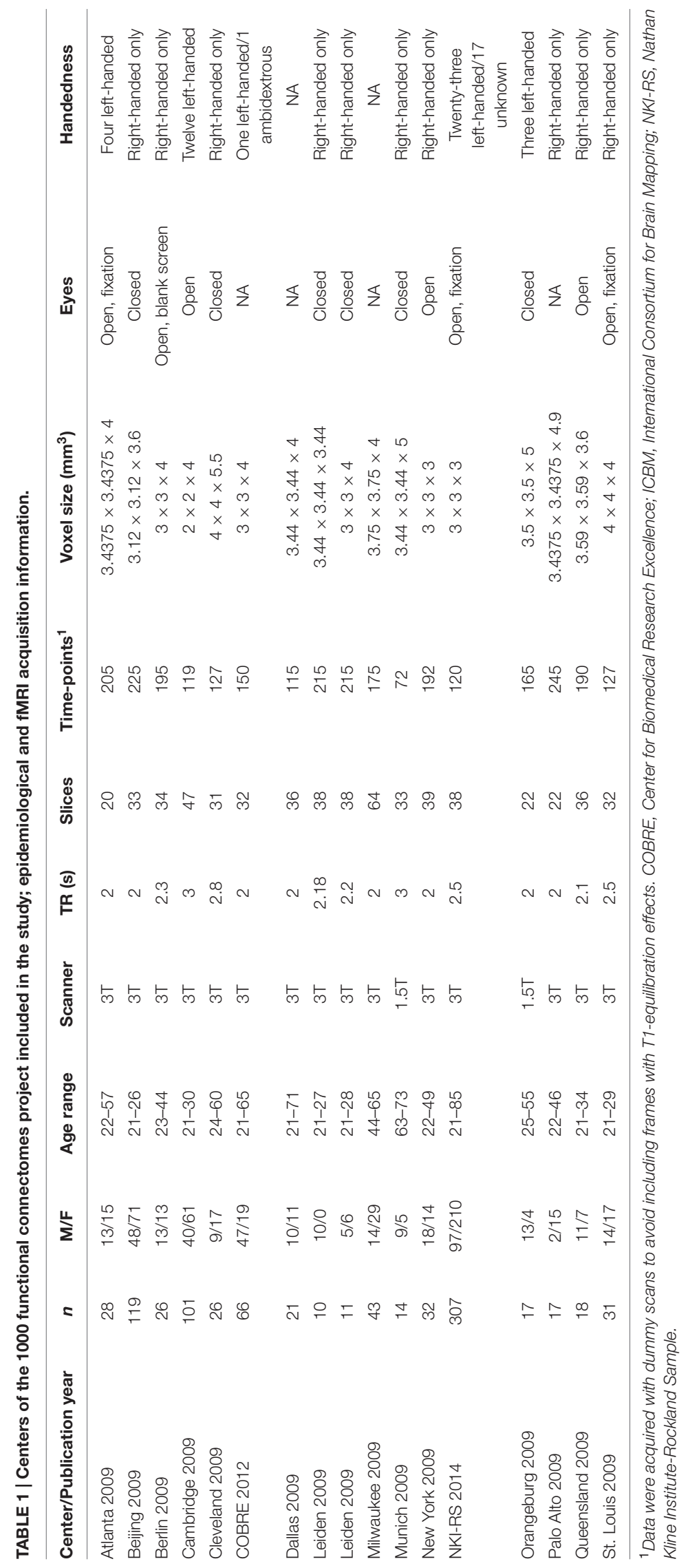


TABLE 2 | Epidemiological data of participants selected for statistical analysis.

\begin{tabular}{|c|c|c|c|c|c|}
\hline Center/Publication year & $n$ & M & $\mathbf{F}$ & Age range & Handedness \\
\hline Atlanta 2009 & 7 & 5 & 2 & $23-57$ & Two left-handed \\
\hline Beijing 2009 & 18 & 7 & 11 & $21-26$ & Right-handed only \\
\hline Berlin 2009 & 5 & 3 & 2 & $26-44$ & Right-handed only \\
\hline Cambridge 2009 & 22 & 8 & 14 & $21-30$ & Three left-handed \\
\hline Cleveland 2009 & 3 & 0 & 3 & $53-60$ & Right-handed only \\
\hline COBRE 2012 & 21 & 15 & 6 & $23-65$ & Right-handed only \\
\hline Dallas 2009 & 11 & 5 & 6 & $21-71$ & NA \\
\hline Leiden 2009 & 4 & 4 & 0 & $21-24$ & Right-handed only \\
\hline Leiden 2009 & 2 & 0 & 2 & $21-22$ & Right-handed only \\
\hline Milwaukee 2009 & 24 & 8 & 16 & $47-65$ & NA \\
\hline Munich 2009 & 14 & 9 & 5 & $63-73$ & Right-handed only \\
\hline New York 2009 & 8 & 4 & 4 & $23-45$ & Right-handed only \\
\hline NKI-RS 2014 & 159 & 46 & 113 & $21-85$ & Fourteen left-handed/5 unknown \\
\hline Orangeburg 2009 & 5 & 5 & 0 & $26-55$ & Right-handed only \\
\hline Palo Alto 2009 & 6 & 0 & 6 & $26-46$ & Right-handed only \\
\hline Queensland 2009 & 2 & 0 & 2 & 26 & Right-handed only \\
\hline St. Louis 2009 & 7 & 3 & 4 & $21-28$ & Right-handed only \\
\hline Total & 318 & 122 & 196 & $21-85$ & 19 left-handed/5 unknown \\
\hline
\end{tabular}

view of previous reports suggesting increased head movement with aging (D’Esposito et al., 1999; Van Dijk et al., 2012), additional head motion parameters were calculated for each participant as proposed by Power et al. (2012) : (1) Framewise displacement (FD), which represents head displacement from volume to volume, was computed as the sum of the first derivative of the six rigid-body motion parameters estimated during standard volume realignment; (2) Delta variation signal (DVARS), which represents the change in BOLD signal intensity from one frame to the next, was computed as the root mean square average of the first derivative of fMRI signals across the entire brain. A standardized version of DVARS was applied according to Nichols (2013). Averaged FD values were below $0.5 \mathrm{~mm}$ for all participants. Averaged standardized DVARS values were between 0.7 and 1.5. Each individual's averaged FD and DVARS values were included in the statistical analysis as described below.

\section{Cortical Thickness Analysis}

To correct for a potential effect of age-related cortical atrophy, we estimated cortical thickness using the Freesurfer image analysis suite, version $5 \cdot 2 \cdot 0^{2}$. Freesurfer uses intensity and continuity information from the entire three dimensional magnetic resonance volume in segmentation and deformation procedures to produce representations of cortical thickness (Dale et al., 1999). Three-dimensional T1-weighted magnetizationprepared rapid acquisition with gradient echo (MPRAGE) scans were used for analysis. Cortical reconstruction and volumetric segmentation were performed for each participant and the boundaries between GM and WM were delineated. Cortical thickness was defined as the closest distance from the GM/WM border to the GM/cerebrospinal fluid border at each vertex

${ }^{2}$ http://surfer.nmr.mgh.harvard.edu/ along the tessellated surface (Fischl and Dale, 2000). Both the ROIs and each participant's surface representation of the cortex were registered to fsaverage, the freesurfer template subject. The measure of cortical thickness was averaged across the vertices that comprise each ROI representation over each participant's surface. These values were then averaged across all ROIs within a network. Each participant's mean cortical thickness per network was included in the statistical analysis as described below.

\section{Statistical Analysis}

Regression modeling was utilized to examine influences on functional connectivity. Averaged $z(r)$ values of node pair correlations within each network were calculated for each participant. These connectivity scores were then analyzed as the dependent variable by a mixed model analysis of covariance (ANCOVA). This modeling method allowed us to examine the effect of a number of factors, and their interactions, while accounting for (1) the effects of covariates on connectivity; (2) systematic differences between test centers; and (3) the influence of individual subjects who each provided data on all networks. Specifically, network, age group, gender and all their factorial interactions served as fixed independent factors; FD, DVARS and cortical thickness served as covariates; test center, assumed to be a random sample from the population of all possible test centers, served as a random effect; and participant, presumed to be part of a random sample of all possible subjects, served as a random factor, necessarily nested in age group, gender and center (utilization of both fixed and random factors makes the model "mixed"). The factor 'age group' had three levels: young (21-40 years), middle-aged (41-60 years), and old (61 years and above), to fit our hypotheses. Gender was included as a factor following prior literature suggesting a gender effect on functional connectivity in general (Allen et al., 2011; Tomasi and Volkow, 2012) and on age-related connectivity 
TABLE 3 | Network regions of interest.

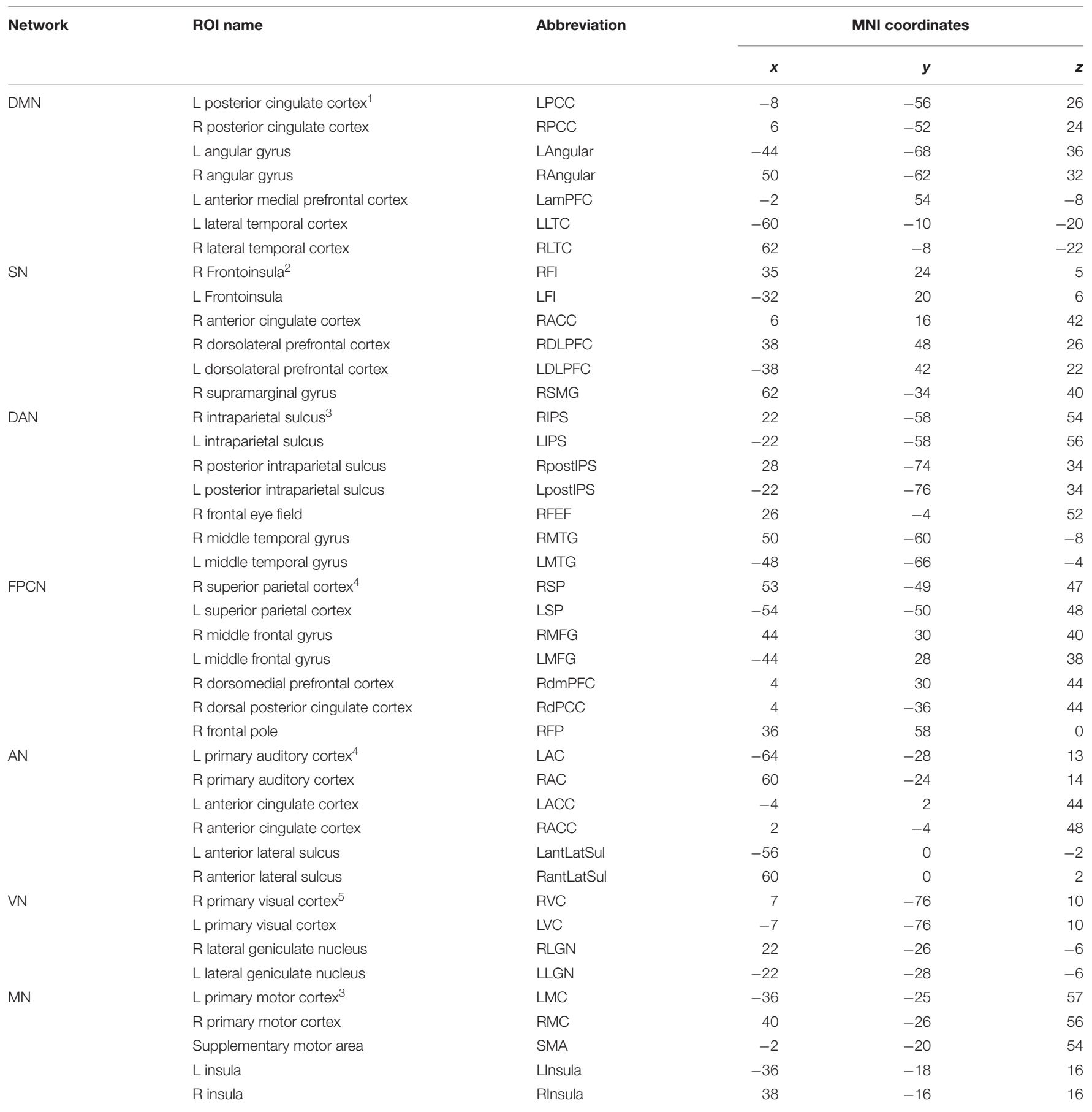

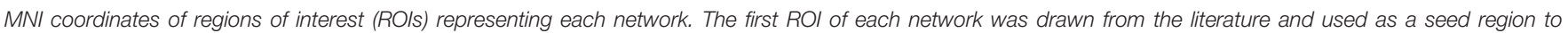
delineate the respective network: (1) Andrews-Hanna et al., 2010. (2) Seeley et al., 2009. (3) Van Dijk et al., 2010. (4) Brier et al., 2012. (5) Dai et al., 2012. L, left; R, right.

changes in particular (Scheinost et al., 2015). We assumed that variability in scanning parameters across centers (scanner magnetic field strength, imaging protocol, image geometry, and scan time) would be sufficiently accounted for by treating center as a random effect. Since an unbalanced model with many factorial interactions could be problematic, 106 participants were randomly selected from the young and middle-aged groups to match the number of individuals within the older age group (Table 2; Supplementary Figure S1). SAS version 9.3, JMP version 11 (both SAS Institute, Cary, NC, USA) and Microsoft Excel 2013 (Microsoft Corp., Redmond, WA, USA) were used for data analysis.

Inspection of ANCOVA diagnostic plots, particularly residual information, did not indicate violation of required normality 
assumptions, so no transformations were applied to the data. Although not a focus of our research, a full treatment of models under mixed modeling generally examines the patterns of relationships (covariance structures) between elements of random effects; when there is no a priori hypothesis for any particular covariance structure, tests of several candidates are run, with the best among them determined by statistical methods, such as information criteria tests. Tests of several candidate covariance structures by SAS PROC MIXED indicated that the variance components covariance structure was most appropriate based on the AICC (corrected Akaike information criterion). This implies that variances associated with each center and each subject were different, and that there was no covariance between either centers or subjects.

The above described model does not provide tests of specific hypotheses of interest regarding the age-group-related connectivity changes. Yet, it does provide the basic information for constructing specific contrasts which are of direct relevance to our hypotheses. Therefore, specific, pre-planned, multiplicitycorrected contrasts were constructed to examine: (1) whether a significant difference emerged within each of the seven networks in the transition from young to middle-aged vs. middle-aged to old; (2) whether these changes in each highorder cognitive network (from young to middle-aged vs. middle-aged to old) were significantly different from equivalent changes in each primary sensory and motor network. The contrasts were multiplicity-corrected (for multiple tests) using the simulation method offered by SAS PROC MIXED. Statistical significance after multiplicity correction was at the level of $p \leq 0.05$.

\section{RESULTS}

\section{Temporal Signature of Age-Related Connectivity Decline}

To study connectivity dynamics across the adult lifespan, connectivity matrices were computed for each age group (young, middle-aged, and old) for the following networks: DMN, SN, DAN, FPCN, AN, VN, and MN. Difference matrices were then calculated for each network between the young and middle-aged (Y-M) and the middle-aged and old (M-O) groups. These comparisons yielded similar results for all high-order cognitive networks (DMN, SN, DAN, and FPCN): Y-M matrices mostly showed significant connectivity reductions, while $\mathrm{M}-\mathrm{O}$ matrices revealed nonsignificant decreases, or a tendency for increased connectivity in some pairs, particularly within the DMN and DAN (Figure 1A). In contrast, the MN typically showed increased connectivity in the transition from young to middle-aged and decreased connectivity in the transition from middle-aged to old. Increased connectivity in the transition to middleage was particularly noted in the connections between the supplementary motor area and the primary motor cortex bilaterally. Within the AN and VN both comparisons yielded significant decrements.
To account for potential sources of bias such as brain atrophy, head motion and varying acquisition parameters, a mixed-model ANCOVA was conducted on a subset of 318 participants of the entire cohort, after equalizing the number of participants in each age group (Table 4). Network and age group showed a highly significant main effect $(p<0.0001)$ and a gender main effect was significant at $p=0.05$. Network $\times$ age group and network $\times$ gender interactions both reached significance $(p<0.0001$ and $p=0.01$, respectively), but network $\times$ age group $\times$ gender interaction did not $(p=0.38)$. Least-squares means (LSM) and standard errors (SE) of the network $\times$ age group interaction are plotted in Figure 1B. These analyses confirmed the findings of the correlation matrices, showing an early decline in connectivity within high-order cognitive networks, predominantly in the DMN and SN. Pre-planned, multiplicity-corrected contrasts of the transition from young to middle-aged against middle-aged to old within each network revealed significant differences for the DMN and MN (Table 5). For the DMN, a significant decrement from young to middleaged was followed by a minor increment from middle-aged to old. The MN showed a completely different pattern of lifelong connectivity changes: an increase from young to middle-age, followed by an equivalent decrease at old age. Contrasts of the transition between the young to middle-aged and middle-aged to old were compared between high-order cognitive networks and primary sensory and motor networks. Pre-planned, multiplicitycorrected comparisons showed a significant difference between the $\mathrm{MN}$ and all other high-order cognitive networks (Table 6).

\section{Spatial Signature of Early Age-Related Connectivity Changes in the DMN and MN}

To anatomically characterize the unique and opposed effects of age on connectivity within the DMN and $\mathrm{MN}$, a twosample $t$-test analysis was used to compare network spatial maps of young vs. middle-aged individuals. Within the DMN, reduced connectivity was shown in middle-aged vs. young participants in several regions of the network, primarily the ventromedial prefrontal cortex (vmPFC), the right lateral temporal cortex (LTC), and the left frontal pole (Figures 2A,C copper-colored regions and Figure 2D). Increased correlation with the LPCC was also observed, mainly within frontal regions bilaterally (Figures 2B,C blue-colored regions and Figure 2E). These regions were found to be associated with the DMN anticorrelated network (Figure 2C, cool colors); therefore, connectivity increments in this case represent reductions in the magnitude of anticorrelation with the LPCC. Within the MN, the comparison between young and middle-aged participants showed decreased connectivity in the latter group in an anterior region of the cerebellum, and increased anticorrelations with regions in the thalamus, basal ganglia and posterior cerebellum (Figures 2F,H copper-colored regions and Figure 2I). Increased connectivity in middle-aged relative to young individuals was noticed mainly in cortical regions of the $\mathrm{MN}$ or border regions adjacent to the MN (Figures 2G,H blue-colored regions and Figure 2J). 


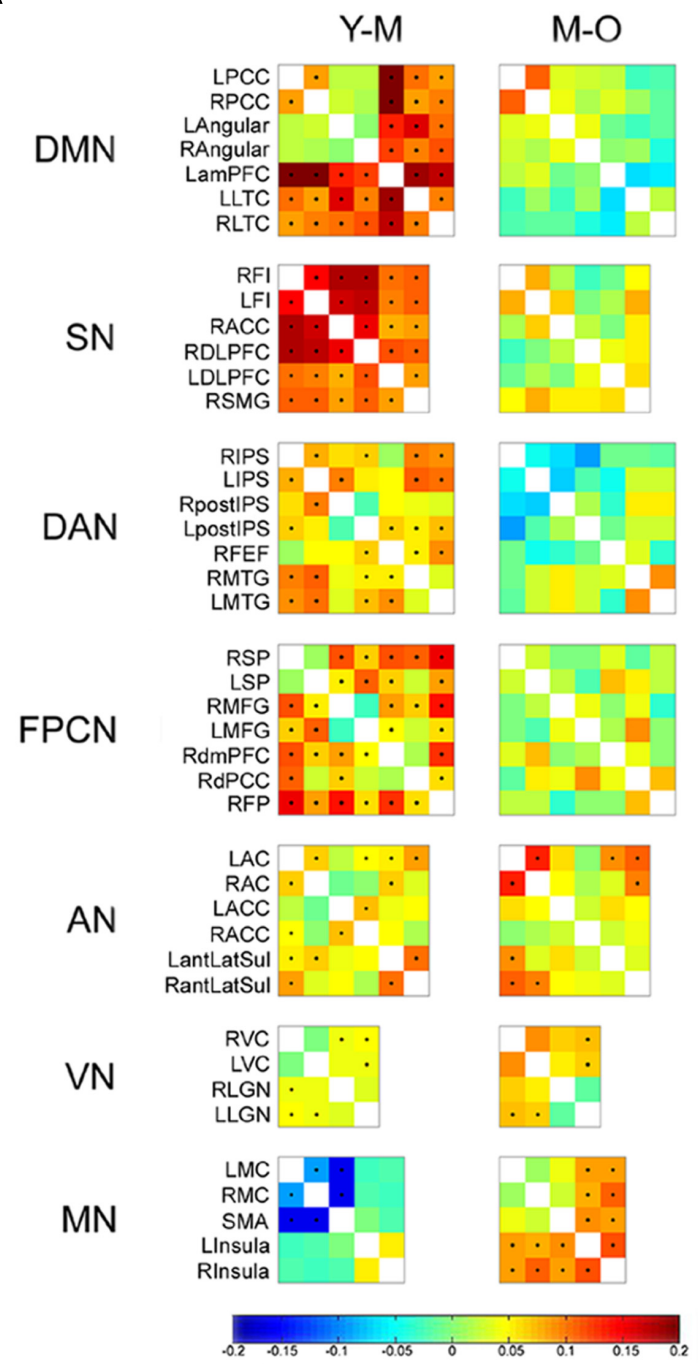

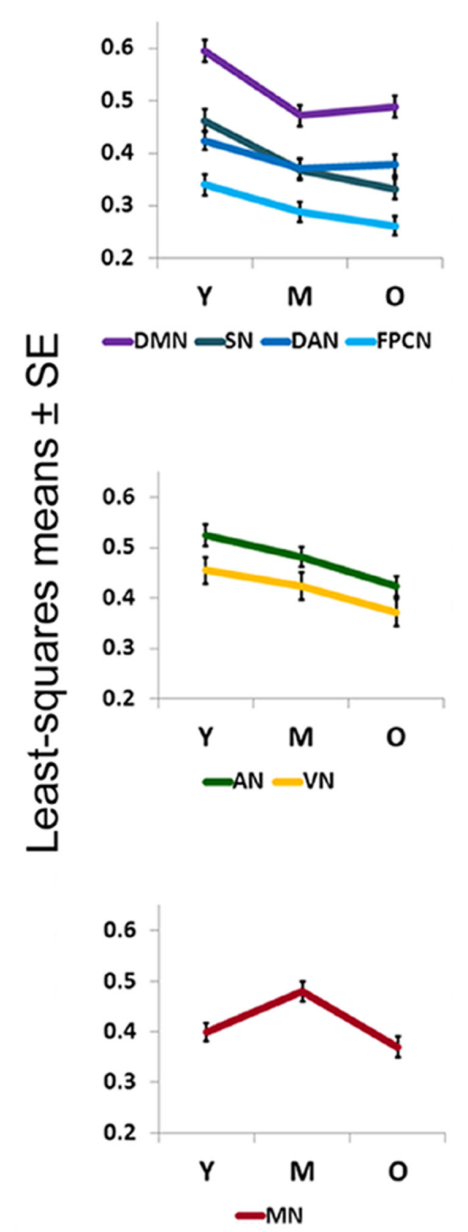

FIGURE 1 | Early age-related connectivity decline in high-order cognitive networks. (A) Difference matrices of young vs. middle-aged (Y-M) and middle-aged vs. old (M-O) participants (entire cohort, $n=887$ ) are shown for each network. Rows and columns of matrices denote the ROls that were chosen to represent each network (see Table 3). Matrix entries represent the age group difference in connectivity strength [z(r) value] for each node pair. Entries marked by a dot survived FDR correction for multiple comparisons. In general, a significant connectivity decline is observed in high-order cognitive networks in the transition between young and middle adulthood. Within the AN and VN significant reductions are observed in some pairs for both transitions, $\mathrm{Y}$ to $\mathrm{M}$ and $\mathrm{M}$ to $\mathrm{O}$. The MN mostly shows early connectivity increments and late connectivity decrements. See Table $\mathbf{3}$ for regions full name. (B) ANCOVA results of the network $\times$ age group interaction [performed on a subset of the entire cohort $(n=318)]$ are presented as least-squares means \pm standard error (SE). Connectivity reduction within high-order cognitive networks is more pronounced in the comparison between $\mathrm{Y}$ to $\mathrm{M}$ than between $\mathrm{M}$ to $\mathrm{O}$. The $\mathrm{AN}$ and $\mathrm{VN}$ show connectivity reduction in both transitions, with a more prominent decline in the transition between $\mathrm{M}$ to $\mathrm{O}$. The $\mathrm{MN}$ shows an early significant connectivity increment followed by a late significant decrement.

\section{Early Age-Related Inter-Network Connectivity Changes}

Connectivity matrices calculated for the three age groups showed connectivity dynamics not only within networks as described above, but also between networks. Most of the internetwork connectivity changes occurred in the transition between the young and middle-aged groups and involved high-order cognitive networks. Early significant reduced connectivity was found between the DMN and MN, SN and AN, and DAN and VN. Early significant increased connectivity was found between the DAN and $\mathrm{MN}, \mathrm{DAN}$ and $\mathrm{AN}, \mathrm{SN}$ and $\mathrm{VN}, \mathrm{AN}$ and $\mathrm{MN}$, parts of the DMN and FPCN, and parts of the AN and $\mathrm{VN}$. Another early change was a decline in anticorrelations: DMNSN, DMN-DAN, and DAN-FPCN. Late changes were much less pronounced. Significant alterations were noticed in only 10 out of 752 node pairs, compared with 203 pairs in the transition from young to middle-age. Late changes included reduced connectivity between the DMN and FPCN and $\mathrm{AN}$ and MN, along with increased connectivity between the $\mathrm{SN}$ and FPCN, SN and $\mathrm{VN}$, and $\mathrm{AN}$ and $\mathrm{VN}$ (Figure 3). 
TABLE 4 | ANCOVA summary.

\begin{tabular}{lccrr}
\hline Effect & Num DF & Den DF & F-value & $\boldsymbol{P}_{\mathbf{r}}>\boldsymbol{F}$ \\
\hline Gender & 1 & 310 & 3.91 & 0.0488 \\
Network & 6 & 1901 & 53.19 & $<0.0001$ \\
Network $\times$ Gender & 6 & 1874 & 2.68 & 0.0135 \\
Age Group & 2 & 325 & 14.59 & $<0.0001$ \\
Age & 2 & 310 & 0.36 & 0.6949 \\
Group $\times$ Gender & 12 & 1874 & 4.36 & $<0.0001$ \\
Network $\times$ Age & 12 & 1871 & 1.08 & 0.376 \\
Group & 1 & & & \\
Network $\times$ Age & 1 & 314 & 24.85 & $<0.0001$ \\
Group $\times$ Gender & 1 & 1873 & 4.18 & 0.0141 \\
DVARS & 1 & & & 0.041 \\
FD & 1 & & &
\end{tabular}

Summary table of ANCOVA for connectivity. Num DF, numerator degrees of freedom; Den DF, denominator degrees of freedom; $F$-value, the F statistic; $P_{r}>F$, the P-value associated with the test of this factor.

TABLE 5 | Pre-planned contrasts between age groups within each network.

\begin{tabular}{ll}
\hline & Multiplicity-adjusted $\boldsymbol{P}$-values \\
\hline DMN & $\mathbf{0 . 0 3 4}$ \\
SN & 0.8786 \\
DAN & 0.8681 \\
FPCN & 0.9993 \\
AN & 0.9999 \\
VN & 0.9993 \\
MN & $\mathbf{0 . 0 0 0 6}$
\end{tabular}

A summary of the pre-planned, multiplicity-corrected contrasts of the transition from young to middle-aged against middle-aged to old within each network.

Statistically significant values are denoted in bold $(p<0.05)$.

TABLE 6 | Pre-planned contrasts between high-order cognitive networks and primary sensory and motor networks.

\begin{tabular}{lc}
\hline & Multiplicity-adjusted P-values \\
\hline DMN vs. MN & $<\mathbf{0 . 0 0 0 1}$ \\
DMN vs. AN & 0.1039 \\
DMN vs. VN & 0.0747 \\
SN vs. MN & $\mathbf{0 . 0 0 0 5}$ \\
SN vs. AN & 0.8835 \\
SN vs. VN & 0.8217 \\
DAN vs. MN & $\mathbf{0 . 0 0 0 5}$ \\
DAN vs. AN & 0.8775 \\
DAN vs. VN & 0.8118 \\
FPCN vs. MN & $\mathbf{0 . 0 0 3 9}$ \\
FPCN vs. AN & 0.9965 \\
FPCN vs. VN & 0.9886
\end{tabular}

A summary of the pre-planned, multiplicity corrected contrasts of the transition between the young to middle-aged and middle-aged to old as compared between high-order cognitive networks and primary sensory and motor networks.

Statistically significant values are denoted in bold $(p<0.05)$.

\section{Global Signal Regression Effect on Study Results}

Reanalysis of the data without GSR yielded similar results (Supplementary Figures S2 and S3). As expected, a general increase in correlations within and between networks was evident (Murphy et al., 2009; Van Dijk et al., 2010; Murphy and Fox, 2016), but this did not affect the major observations of the study. Specifically, the within network correlation pattern did not change. Age group difference matrices revealed greater reduction in the transition from $\mathrm{Y}$ to $\mathrm{M}$ than from $\mathrm{M}$ to $\mathrm{O}$ for the DMN, SN, and FPCN. The MN showed increased connectivity from $\mathrm{Y}$ to $\mathrm{M}$ followed by a reduction for $\mathrm{O}$. The $\mathrm{AN}$ and $\mathrm{VN}$ showed reductions in both transitions, but $\mathrm{Y}$ to $\mathrm{M}$ reduction in the AN was much less significant relative to results with GSR. The observation of a less significant age-related reduction when avoiding GSR, particularly within the DAN and AN from Y to $\mathrm{M}$, might be related to high correlation between the signal of these networks and the global signal. The global signal has been shown to highly correlate with signals of the visual, auditory and somatosensory networks (Fox et al., 2009). In our study the DAN was positively correlated with these three non-cognitive networks.

Between network correlations appeared to be affected more markedly by GSR removal. However, this mainly stemmed from the expected disappearance of anticorrelations (Fox et al., 2009; Murphy et al., 2009), while the direction of change between age groups was mostly kept, with the exception of the early decrement in DMN-MN and the late increment in AN-VN. Moreover, the finding that most of the inter-network connectivity alterations occur in the transition from young to middle-age rather than from middle-age to old was even accentuated by omitting GSR.

\section{DISCUSSION}

The present findings indicate that functional connectivity (FC) decline in high-order cognitive networks is already evident by middle-age, as expressed by fcMRI measures. The time course of FC alterations within a motor network is characterized by an early increment followed by a decrement. Finally, most inter-network connectivity changes occur in the transition from young to middle adulthood, highlighting that several potentially meaningful FC changes onset early in the adult lifespan.

Growing evidence now suggests that age-related cognitive decline is associated with FC alterations within large-scale brain networks (Andrews-Hanna et al., 2007; Damoiseaux et al., 2008; Ferreira and Busatto, 2013). The DMN has been the focus of much of this literature, most studies reported FC decline with age and associated FC decline with reduced cognitive performance (e.g., Andrews-Hanna et al., 2007; Damoiseaux et al., 2008; Ferreira and Busatto, 2013; Vidal-Piñeiro et al., 2014). More recently, age-related connectivity decreases have also been noted in other ICNs (Andrews-Hanna et al., 2007; Onoda et al., 2012; Tomasi and Volkow, 2012; Ferreira and Busatto, 2013; Geerligs et al., 2015). Importantly, most of the above mentioned studies directly compared young and old adults, while only a few studied young and middle-aged individuals and 


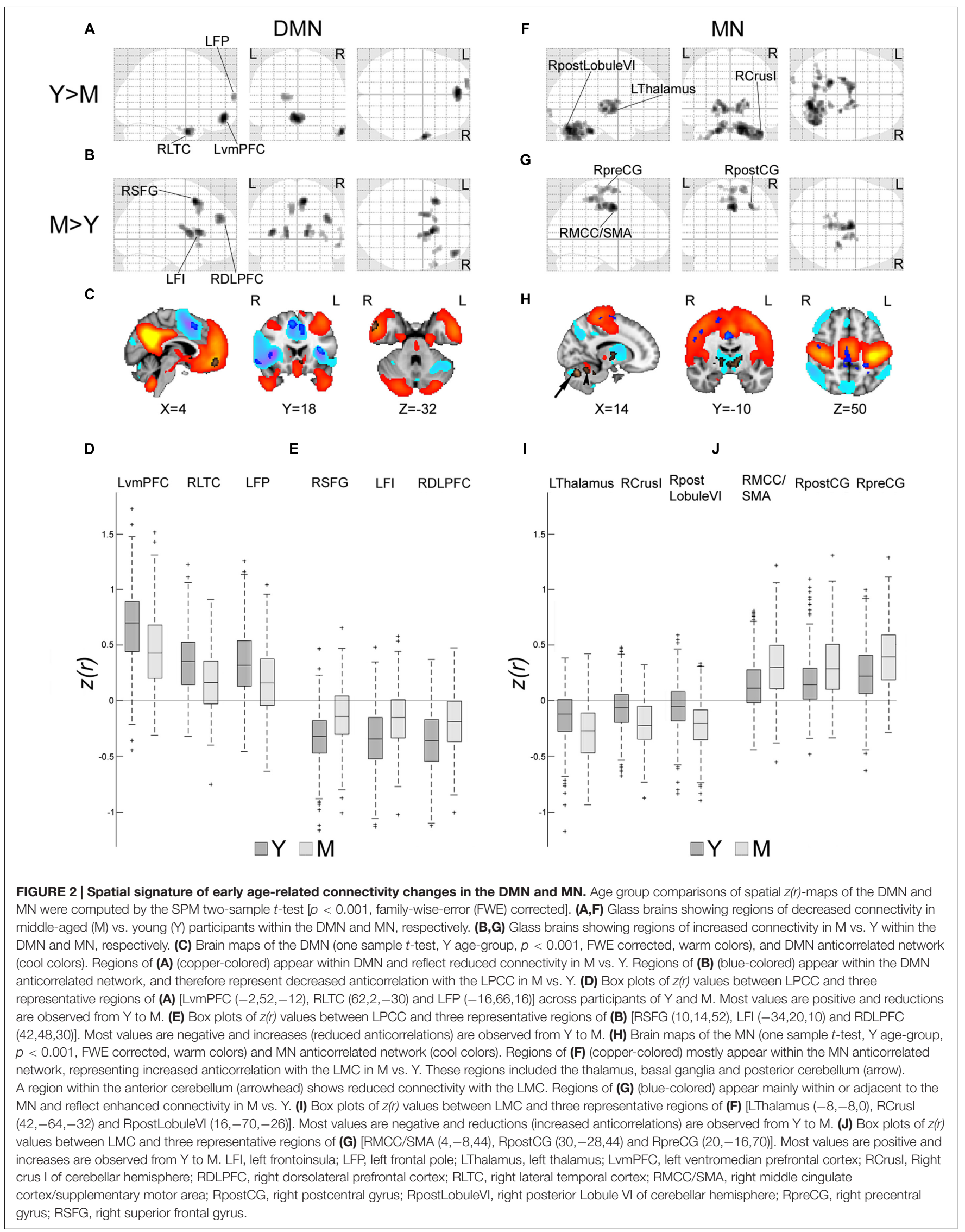



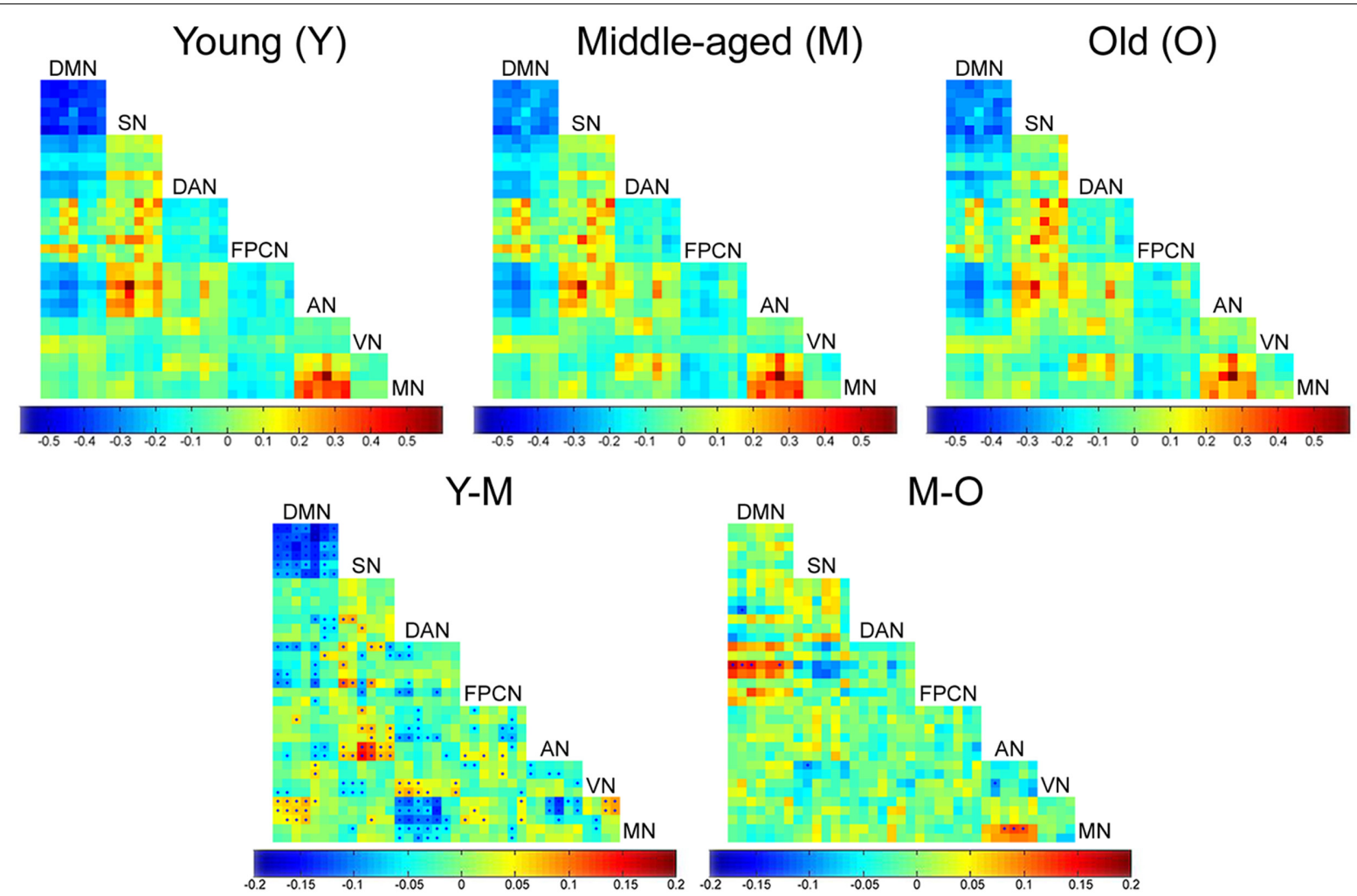

FIGURE 3 | Age-related inter-network connectivity changes. Inter-network connectivity matrices of the young ( $\mathrm{Y}$ ), middle-aged (M) and old ( $\mathrm{O}$ ) groups (upper row). Rows and columns denote the ROls that were chosen to represent each network (see Table 3). Matrix entries represent connectivity strength between each two nodes (z(r) value). Difference matrices of the $\mathrm{Y}$ vs. M and M vs. O groups (lower row). Matrix entries represent age-group difference in connectivity strength for each node pair. Entries marked by a dot survived FDR correction for multiple comparisons. Most significant age-related changes in inter-network connectivity occurred in the transition between $\mathrm{Y}$ and $\mathrm{M}$ groups.

actually reported diminished FC in middle-adulthood (Bluhm et al., 2008; Allen et al., 2011; Evers et al., 2012). Here, FC decline by middle-age is observed in an extensive cohort of individuals within the DMN, SN, DAN, and PFCN. This early FC decline accords with previously suggested trajectories of cognitive and structural deterioration with aging (Buckner, 2004; Park and Reuter-Lorenz, 2009; Giorgio et al., 2010; Chen et al., 2013). Furthermore, several recent studies explicitly underscored a detectable cognitive decline by middle-age in both humans (Singh-Manoux et al., 2012; Ferreira et al., 2015) and animals (Moore et al., 2006; Stouffer and Yoder, 2011; Shoji et al., 2016), supporting the view that connectivity decreases in specific functional brain systems may be associated with particular behavioral changes (Andrews-Hanna et al., 2007; Onoda et al., 2012).

Even more remarkable is the fact that within high-order cognitive networks young to middle-age FC decline is more pronounced than the changes observed between middle-aged and older adults. While this finding conforms with earlier structural connectivity findings (Salat et al., 2005; Giorgio et al., 2010) as well as with age-related alterations in genetic, biochemical, and neurophysiological variables (Antonini et al., 1993; Kuhn et al., 1996; Mozley et al., 1996; Sheline et al., 2002; Lu et al., 2004; Hattiangady et al., 2005; Rex et al., 2005; Lynch et al., 2006;
Hamilton et al., 2013; Aliper et al., 2015), it appears inconsistent with the progressive course of cognitive decline. Although the source for this lifespan FC dynamics cannot be determined by the current study, compensation and dedifferentiation theories might be proposed to account for the apparent late FC plateau. Park and Reuter-Lorenz (2009) suggested in their "scaffolding theory of aging and cognition" (STAC) that although the brain is subjected to multiple neural challenges throughout the lifespan, adaptive compensatory processes help it maintain homeostatic cognitive function up to a certain point. The absence of further FC decline and even connectivity increment in several node pairs in older age might be related to such compensatory mechanisms, as prudent observation of a few behavioral studies seems to reveal a hitherto unreported cognitive plateau around middleage (Villardita et al., 1985; Park et al., 1996; Buckner, 2004; Park and Payer, 2006). Dedifferentiation, the loss of functional specialization in brain activity, is an alternative theory commonly proposed to account for cognitive aging (Park and ReuterLorenz, 2009). One model which was proposed to represent dedifferentiation is the hemispheric asymmetry reduction in older adults (HAROLD) (Cabeza et al., 2002; Dolcos et al., 2002; Li et al., 2009). In line with this model and in accordance with our findings, Zuo et al. (2010) showed that FC between geometrically corresponding interhemispheric regions follows a 
quadratic U-shaped trajectory across the lifespan with a turning point in middle-age in heteromodal regions. Future insights into the physiological origins of BOLD-based FC and their agerelated changes (e.g., dopaminergic transmission; Ferreira and Busatto, 2013) as well as future studies concurrently evaluating FC and cognitive performance may inform on the underlying mechanisms of the lifetime FC variations observed here.

In addition to temporal aspects of connectivity decline, spatial features should be considered. Our study supports the view that different brain systems are differentially affected by aging. Here, the MN showed a significantly different FC time course compared with high-order cognitive networks (early FC increment followed by a late decrement). Age-related changes in FC within the MN have been studied only rarely and results are conflicting with both decreases (Wu et al., 2007) and increases (Tomasi and Volkow, 2012; Solesio-Jofre et al., 2014) reported. However, consistent with our finding, a previous analysis of brain structural covariance networks (Li et al., 2013) uncovered a difference between motor and high-order cognitive networks. Li et al. (2013) report that non-motor networks demonstrate a distributed topology in the young group, shrinkage into a more localized topology in the middle-aged group, and maintained localized topology in the older group, while the MN shows increased spatial distribution in middle-age and decreased distribution in older participants. Moreover, a recent study by Song et al. (2014) reported an equivalent opposing effect of aging on FC within the DMN and a sensorimotor network.

The observed difference between the motor and high-order cognitive networks is in line with the "last in, first out" hypothesis. Moreover, differential vulnerability to aging is also noted within the same network. Interestingly, the vmPFC, LTC and frontal pole, here showing maximal connectivity reduction among DMN regions, were previously reported as being regions of advanced age-related atrophy and greater expansion during evolution (Hill et al., 2010; Bludau et al., 2014; Fjell et al., 2014). As for the $\mathrm{MN}$, the reduced connectivity in an anterior cerebellar region, probably associated with motor function (Bernard et al., 2013), is consistent with previous reports of reduced cortico-cerebellar connectivity with aging (Bernard et al., 2013; Bernard and Seidler, 2014). The concurrent increased connectivity in cortical MN regions is consistent with the hypothesis that shorter connections are enhanced in older individuals, while longer connections are diminished (Rowe et al., 2006). Other early changes observed in the MN are probably due to increased anticorrelations of subcortical regions with the LMC.

Age-related alterations in interrelations between networks represent another central finding of the current study. Most prominent alterations were increased correlations (e.g., DAN$\mathrm{MN}$ ) and decreased anticorrelations (e.g., DMN-SN) between networks in the transition from young to middle adulthood. Our observations strongly support accumulating evidence for enhanced communication between functional networks with aging (Chan et al., 2014; Geerligs et al., 2015; Turner and Spreng, 2015; Gallen et al., 2016; Spreng et al., 2016; Tsvetanov et al., 2016). Increased correlations and decreased anticorrelations between networks both reflect reduced selectivity and specificity in the brain's intrinsic functional architecture. These changes have been associated with compromised cognitive performance (Kelly et al., 2008; Hampson et al., 2010; Antonenko and Floel, 2014; Chan et al., 2014; Tsvetanov et al., 2016) and hence comply with the dedifferentiation theory of aging; yet, a role in compensation has also been suggested (Gallen et al., 2016; Grady et al., 2016). It should be mentioned that GSR used as a preprocessing step in our study has been reported to artifactually enhance anticorrelations between networks (Fox et al., 2009; Murphy et al., 2009). However, a repeated analysis without GSR confirmed age-related reduced anticorrelation between the DMN and SN (Supplementary Figure S3). This finding is consistent with recent aging studies omitting GSR (Keller et al., 2015; Spreng et al., 2016) and supports previous literature suggesting neural rather than artifactual origin of anticorrelations (Chang and Glover, 2009; Fox et al., 2009; Carbonell et al., 2011; Chai et al., 2012; Keller et al., 2013; Power et al., 2014). Additionally, in line with a few previous reports (Allen et al., 2011; Onoda et al., 2012), our analysis also reveals diminished between-network connectivity with aging for some network pairs. Undoubtedly, the complexity of age-related changes in integration of information between networks warrants further investigation. The importance of the present findings is in pointing out decreased segregation of brain systems by middle-age.

The use of a dataset such as the ' 1000 Functional Connectomes' Project inherently imposes a source of variability among participants in terms of demographic parameters, cognitive intactness, arousal level, eye opening condition, head movement and technical acquisition parameters, features that have been reported to affect resting-state correlations (Power et al., 2012; Van Dijk et al., 2010, 2012). The statistical analysis was designed to account for these potential biases, as far as the data allowed. Inclusion of participants of different age groups further augments variability in terms of resting metabolic rate (Peng et al., 2014), resting cerebral blood flow (Lu et al., 2011), vascular $\mathrm{CO}_{2}$ reactivity (Lu et al., 2011; Murphy et al., 2013); blood pressure (AlGhatrif et al., 2017); and hemoglobin concentration (Patel, 2008) all known to affect BOLD fMRI signal and/or the coupling of neural activity to the BOLD signal (D'Esposito et al., 1999; Levin et al., 2001; Patel et al., 2012; Murphy et al., 2013; Mark et al., 2015). Nevertheless, the uneven effect of aging on large-scale brain networks (e.g., DMN vs. MN), emphasized here and elsewhere (Song et al., 2014; Marstaller et al., 2015), probably indicates that age-related FC alterations cannot be merely attributed to changes in neurovascular coupling. Moreover, the GSR step applied in data preprocessing is expected to lessen the confounding effects of the above mentioned physiological factors as well as of cardiac and respiratory cycles (Van Dijk et al., 2010), which have not been directly measured. Though a matter of long-standing debate (Fox et al., 2009; Murphy et al., 2009; Murphy and Fox, 2016), GSR seemed warranted under the current study due to several important reasons: it was shown to enhance the detection of network-specific seed-based correlations (Fox et al., 2009), which are at the core interest of this work; reduce motion and hardware artifacts (Power et al., 2016), an important advantage when evaluating a large 
multi-center dataset; and enhance the neuronal-hemodynamic correspondence (Keller et al., 2013). Taking into consideration the interpretive complexity imposed by GSR (Murphy and Fox, 2016), we repeated the analyses without GSR, confirming that the study's main outcomes are present. Another limitation of this study is the absence of data regarding WM hyperintensity burden which has been shown to increase with age (Nyquist et al., 2015) and affect FC (Liang et al., 2016). As both FC decrements and increments have been reported in the presence of WM lesions (Liang et al., 2016), it is difficult to appreciate this parameter's influence on our results. Finally, the absence of cognitive data for this cohort limits the ability to contemplate regarding the mechanisms underlying current observations. Future single-site longitudinal studies combining detailed analysis of behavioral performance, elaborated demographic information, laboratory data, structural and functional imaging data, as well as measures to control for non-neuronal physiological parameters, might better address the above mentioned confounds and expand the scope of our findings.

In summary, our results confirm the hypothesis that normal brain aging involves reorganization of large-scale functional brain systems. Furthermore, it underscores the early occurrence of FC alterations where high-order cognitive networks are involved. In an era in which we seek ways of augmenting cognition throughout healthy aging, deferring the onset of connectivity decline, slowing down its pace and prolonging the proposed subsequent plateau should be considered potential targets for intervention.

\section{ETHICS STATEMENT}

The imaging data of the current study were obtained from 17 research sites through the online dataset of the International Neuroimaging Data-sharing Initiative (INDI). Each center's ethics committee approved submission of de-identified data as detailed in the NITRC: '1000 Functional Connectomes' Project

\section{REFERENCES}

AlGhatrif, M., Wang, M., Fedorova, O. V., Bagrov, A. Y., and Lakatta, E. G. (2017). The pressure of aging. Med. Clin. North Am. 101, 81-101. doi: 10.1016/j.mcna. 2016.08.006

Aliper, A. M., Csoka, A. B., Buzdin, A., Jetka, T., Roumiantsev, S., Moskalev, A., et al. (2015). Signaling pathway activation drift during aging: HutchinsonGilford Progeria Syndrome fibroblasts are comparable to normal middle-age and old-age cells. Aging (Albany NY) 7, 26-37. doi: 10.18632/aging.100717

Allen, E. A., Erhardt, E. B., Damaraju, E., Gruner, W., Segall, J. M., Silva, R. F., et al. (2011). A baseline for the multivariate comparison of resting-state networks. Front. Syst. Neurosci. 5:2. doi: 10.3389/fnsys.2011.00002

Andrews-Hanna, J. R., Reidler, J. S., Sepulcre, J., Poulin, R., and Buckner, R. L. (2010). Functional-anatomic fractionation of the brain's default network. Neuron 65, 550-562. doi: 10.1016/j.neuron.2010.02.005

Andrews-Hanna, J. R., Snyder, A. Z., Vincent, J. L., Lustig, C., Head, D., Raichle, M. E., et al. (2007). Disruption of large-scale brain systems in advanced aging. Neuron 56, 924-935. doi: 10.1016/j.neuron.2007.10.038

Antonenko, D., and Floel, A. (2014). Healthy aging by staying selectively connected: a mini-review. Gerontology 60, 3-9. doi: 10.1159/000354376 site (http://fcon_1000.projects.nitrc.org/). The institutional review board of Rambam healthcare campus approved the receipt and analysis of these data.

\section{AUTHOR CONTRIBUTIONS}

TS-T, NB, AE, JA-P, and IK contributed to the conception and design of this work. TS-T, NB, and IK collected, analyzed, and interpreted the data. RP contributed to data analysis. ES designed and performed the statistical analyses. TS-T, NB, ES, JA-P, and IK contributed to in-depth discussions about methods and results. TS-T and IK wrote the paper. All authors revised and approved the manuscript.

\section{FUNDING}

Support was provided by the Israel Science Foundation (225/11), the Crown Family Foundation and the Allen and Jewel Prince Center for Neurodegenerative Processes of the Brain. This research was conducted while IK was a new investigator as an Alzheimer's Disease Grant recipient from the American Federation for Aging Research.

\section{ACKNOWLEDGMENT}

We thank Dr. Alexandra Kavushansky, Dr. Noga Adler, Eyal Bergmann and Guy Yona for their helpful comments regarding the data analysis.

\section{SUPPLEMENTARY MATERIAL}

The Supplementary Material for this article can be found online at: http://journal.frontiersin.org/article/10.3389/fnagi. 2016.00330/full\#supplementary-material

Antonini, A., Leenders, K. L., Reist, H., Thomann, R., Beer, H. F., and Locher, J. (1993). Effect of age on D2 dopamine receptors in normal human brain measured by positron emission tomography and 11Craclopride. Arch. Neurol. 50, 474-480. doi: 10.1001/archneur.1993.005400500 26010

Bäckman, L., Ginovart, N., Dixon, R. A., Wahlin, T. B., Wahlin, A., Halldin, C., et al. (2000). Age-related cognitive deficits mediated by changes in the striatal dopamine system. Am. J. Psychiatry 157, 635-637. doi: 10.1176/ajp.157. 4.635

Bäckman, L., Lindenberger, U., Li, S. C., and Nyberg, L. (2010). Linking cognitive aging to alterations in dopamine neurotransmitter functioning: recent data and future avenues. Neurosci. Biobehav. Rev. 34, 670-677. doi: 10.1016/j.neubiorev. 2009.12.008

Bäckman, L., Nyberg, L., Lindenberger, U., Li, S. C., and Farde, L. (2006). The correlative triad among aging, dopamine, and cognition: current status and future prospects. Neurosci. Biobehav. Rev. 30, 791-807. doi: 10.1016/j. neubiorev.2006.06.005

Belcher, A. M., Yen, C. C., Stepp, H., Gu, H., Lu, H., Yang, Y., et al. (2013). Largescale brain networks in the awake, truly resting marmoset monkey. J. Neurosci. 33, 16796-16804. doi: 10.1523/JNEUROSCI.3146-13.2013 
Benjamini, Y., and Yekutieli, D. (2001). The control of the false discovery rate in multiple testing under dependency. Ann. Stat. 29, 1165-1188. doi: 10.1186/ 1471-2105-9-114

Bergfield, K. L., Hanson, K. D., Chen, K., Teipel, S. J., Hampel, H., Rapoport, S. I., et al. (2010). Age-related networks of regional covariance in MRI gray matter: reproducible multivariate patterns in healthy aging. Neuroimage 49, 1750-1759. doi: 10.1016/j.neuroimage.2009.09.051

Bernard, J. A., Peltier, S. J., Wiggins, J. L., Jaeggi, S. M., Buschkuehl, M., Fling, B. W., et al. (2013). Disrupted cortico-cerebellar connectivity in older adults. Neuroimage 83, 103-119. doi: 10.1016/j.neuroimage.2013. 06.042

Bernard, J. A., and Seidler, R. D. (2014). Moving forward: age effects on the cerebellum underlie cognitive and motor declines. Neurosci. Biobehav. Rev. 42C, 193-207. doi: 10.1016/j.neubiorev.2014.02.011

Betzel, R. F., Byrge, L., He, Y., Goñi, J., Zuo, X. N., and Sporns, O. (2014). Changes in structural and functional connectivity among resting-state networks across the human lifespan. Neuroimage 102, 345-357. doi: 10.1016/j.neuroimage.2014. 07.067

Bludau, S., Eickhoff, S. B., Mohlberg, H., Caspers, S., Laird, A. R., Fox, P. T., et al. (2014). Cytoarchitecture, probability maps and functions of the human frontal pole. Neuroimage 93, 260-275. doi: 10.1016/j.neuroimage.2013.05.052

Bluhm, R. L., Osuch, E. A., Lanius, R. A., Boksman, K., Neufeld, R. W., Theberge, J., et al. (2008). Default mode network connectivity: effects of age, sex, and analytic approach. Neuroreport 19, 887-891. doi: 10.1097/WNR.0b013e328300ebbf

Braga, R. M., and Leech, R. (2015). Echoes of the brain: local-scale representation of whole-brain functional networks within transmodal cortex. Neuroscientist 21, 540-551. doi: 10.1177/1073858415585730

Brier, M. R., Thomas, J. B., Snyder, A. Z., Benzinger, T. L., Zhang, D., Raichle, M. E., et al. (2012). Loss of intranetwork and internetwork resting state functional connections with Alzheimer's disease progression. J. Neurosci. 32, 8890-8899. doi: 10.1523/JNEUROSCI.5698-11.2012

Buckner, R. L. (2004). Memory and executive function in aging and AD: multiple factors that cause decline and reserve factors that compensate. Neuron 44, 195-208. doi: 10.1016/j.neuron.2004.09.006

Buckner, R. L., Krienen, F. M., and Yeo, B. T. (2013). Opportunities and limitations of intrinsic functional connectivity MRI. Nat. Neurosci. 16, 832-837. doi: 10. 1038/nn.3423

Cabeza, R., Anderson, N. D., Locantore, J. K., and McIntosh, A. R. (2002). Aging gracefully: compensatory brain activity in high-performing older adults. Neuroimage 17, 1394-1402. doi: 10.1006/nimg.2002.1280

Carbonell, F., Bellec, P., and Shmuel, A. (2011). Global and system-specific restingstate fMRI fluctuations are uncorrelated: principal component analysis reveals anti-correlated networks. Brain Connect. 1, 496-510. doi: 10.1089/brain.2011. 0065

Chai, X. J., Castañón, A. N., Ongür, D., and Whitfield-Gabrieli, S. (2012). Anticorrelations in resting state networks without global signal regression. Neuroimage 59, 1420-1428. doi: 10.1016/j.neuroimage.2011.08.048

Chan, M. Y., Park, D. C., Savalia, N. K., Petersen, S. E., and Wig, G. S. (2014). Decreased segregation of brain systems across the healthy adult lifespan. Proc. Natl. Acad. Sci. U.S.A. 111, E4997-E5006. doi: 10.1073/pnas.1415122111

Chang, C., and Glover, G. H. (2009). Effects of model-based physiological noise correction on default mode network anti-correlations and correlations. Neuroimage 47, 1448-1459. doi: 10.1016/j.neuroimage.2009.05.012

Chen, X., Errangi, B., Li, L., Glasser, M. F., Westlye, L. T., Fjell, A. M., et al. (2013). Brain aging in humans, chimpanzees (Pan troglodytes), and rhesus macaques (Macaca mulatta): magnetic resonance imaging studies of macroand microstructural changes. Neurobiol. Aging 34, 2248-2260. doi: 10.1016/j. neurobiolaging.2013.03.028

Dai, H., Morelli, J. N., Ai, F., Yin, D., Hu, C., Xu, D., et al. (2012). Restingstate functional MRI: functional connectivity analysis of the visual cortex in primary open-angle glaucoma patients. Hum. Brain Mapp. 34, 2455-2463. doi: 10.1002/hbm.22079

Dale, A. M., Fischl, B., and Sereno, M. I. (1999). Cortical surface-based analysis. I. Segmentation and surface reconstruction. Neuroimage 9, 179-194.

Damoiseaux, J. S., Beckmann, C. F., Arigita, E. J., Barkhof, F., Scheltens, P., Stam, C. J., et al. (2008). Reduced resting-state brain activity in the "default network" in normal aging. Cereb. Cortex 18, 1856-1864. doi: 10.1093/cercor/bhm207
Damoiseaux, J. S., Rombouts, S. A., Barkhof, F., Scheltens, P., Stam, C. J., Smith, S. M., et al. (2006). Consistent resting-state networks across healthy subjects. Proc. Natl. Acad. Sci. U.S.A. 103, 13848-13853. doi: 10.1073/pnas.0601417103

D’Esposito, M., Zarahn, E., Aguirre, G. K., and Rypma, B. (1999). The effect of normal aging on the coupling of neural activity to the bold hemodynamic response. Neuroimage 10, 6-14. doi: 10.1006/nimg.1999.0444

Dolcos, F., Rice, H. J., and Cabeza, R. (2002). Hemispheric asymmetry and aging: right hemisphere decline or asymmetry reduction. Neurosci. Biobehav. Rev. 26, 819-825. doi: 10.1016/S0149-7634(02)00068-4

Erixon-Lindroth, N., Farde, L., Wahlin, T. B., Sovago, J., Halldin, C., and Bäckman, L. (2005). The role of the striatal dopamine transporter in cognitive aging. Psychiatry Res. 138, 1-12. doi: 10.1016/j.pscychresns.2004.09.005

Evers, E. A., Klaassen, E. B., Rombouts, S. A., Backes, W. H., and Jolles, J. (2012). The effects of sustained cognitive task performance on subsequent resting state functional connectivity in healthy young and middle-aged male schoolteachers. Brain Connect. 2, 102-112. doi: 10.1089/brain.2011.0060

Ferreira, D., Correia, R., Nieto, A., Machado, A., Molina, Y., and Barroso, J. (2015). Cognitive decline before the age of 50 can be detected with sensitive cognitive measures. Psicothema 27, 216-222. doi: 10.7334/psicothema2014.192

Ferreira, D., Molina, Y., Machado, A., Westman, E., Wahlund, L. O., Nieto, A., et al. (2014). Cognitive decline is mediated by gray matter changes during middle age. Neurobiol. Aging 35, 1086-1094. doi: 10.1016/j.neurobiolaging.2013. 10.095

Ferreira, L. K., and Busatto, G. F. (2013). Resting-state functional connectivity in normal brain aging. Neurosci. Biobehav. Rev. 37, 384-400. doi: 10.1016/j. neubiorev.2013.01.017

Fischl, B., and Dale, A. M. (2000). Measuring the thickness of the human cerebral cortex from magnetic resonance images. Proc. Natl. Acad. Sci. U.S.A. 97, 11050-11055. doi: 10.1073/pnas.200033797

Fjell, A. M., McEvoy, L., Holland, D., Dale, A. M., and Walhovd, K. B. (2014). What is normal in normal aging? Effects of aging, amyloid and Alzheimer's disease on the cerebral cortex and the hippocampus. Prog. Neurobiol. 117, 20-40. doi: 10.1016/j.pneurobio.2014.02.004

Fox, M. D., and Raichle, M. E. (2007). Spontaneous fluctuations in brain activity observed with functional magnetic resonance imaging. Nat. Rev. Neurosci. 8, 700-711. doi: 10.1038/nrn2201

Fox, M. D., Snyder, A. Z., Vincent, J. L., Corbetta, M., Van Essen, D. C., and Raichle, M. E. (2005). The human brain is intrinsically organized into dynamic, anticorrelated functional networks. Proc. Natl. Acad. Sci. U.S.A. 102, 9673-9678. doi: 10.1073/pnas.0504136102

Fox, M. D., Zhang, D., Snyder, A. Z., and Raichle, M. E. (2009). The global signal and observed anticorrelated resting state brain networks. J. Neurophysiol. 101, 3270-3283. doi: 10.1152/jn. 90777.2008

Gallen, C. L., Turner, G. R., Adnan, A., and D’Esposito, M. (2016). Reconfiguration of brain network architecture to support executive control in aging. Neurobiol. Aging 44, 42-52. doi: 10.1016/j.neurobiolaging.2016.04.003

Ge, Y., Grossman, R. I., Babb, J. S., Rabin, M. L., Mannon, L. J., and Kolson, D. L. (2002). Age-related total gray matter and white matter changes in normal adult brain. Part I: volumetric MR imaging analysis. AJNR Am. J. Neuroradiol. 23, 1327-1333.

Geerligs, L., Renken, R. J., Saliasi, E., Maurits, N. M., and Lorist, M. M. (2015). A brain-wide study of age-related changes in functional connectivity. Cereb. Cortex 25, 1987-1999. doi: 10.1093/cercor/bhu012

Giorgio, A., Santelli, L., Tomassini, V., Bosnell, R., Smith, S., De Stefano, N., et al. (2010). Age-related changes in grey and white matter structure throughout adulthood. Neuroimage 51, 943-951. doi: 10.1016/j.neuroimage.2010.03.004

Good, C. D., Johnsrude, I. S., Ashburner, J., Henson, R. N., Friston, K. J., and Frackowiak, R. S. (2001). A voxel-based morphometric study of ageing in 465 normal adult human brains. Neuroimage 14, 21-36. doi: 10.1006/nimg.2001. 0786

Grady, C., Sarraf, S., Saverino, C., and Campbell, K. (2016). Age differences in the functional interactions among the default, frontoparietal control, and dorsal attention networks. Neurobiol. Aging 41, 159-172. doi: 10.1016/j. neurobiolaging.2016.02.020

Hamilton, L. K., Joppé, S. E. M., Cochard, L., and Fernandes, K. J. (2013). Aging and neurogenesis in the adult forebrain: what we have learned and where we should go from here. Eur. J. Neurosci. 37, 1978-1986. doi: 10.1111/ejn.12207 
Hampson, M., Driesen, N., Roth, J. K., Gore, J. C., and Constable, R. T. (2010). Functional connectivity between task-positive and task-negative brain areas and its relation to working memory performance. Magn. Reson. Imaging 28, 1051-1057. doi: 10.1016/j.mri.2010.03.021

Hattiangady, B., Rao, M. S., Shetty, G. A., and Shetty, A. K. (2005). Brain-derived neurotrophic factor, phosphorylated cyclic AMP response element binding protein and neuropeptide $\mathrm{Y}$ decline as early as middle age in the dentate gyrus and CA1 and CA3 subfields of the hippocampus. Exp. Neurol. 195, 353-371. doi: 10.1016/j.expneurol.2005.05.014

Hedden, T., and Gabrieli, J. D. (2004). Insights into the ageing mind: a view from cognitive neuroscience. Nat. Rev. Neurosci. 5, 87-96. doi: 10.1038/nrn1323

Hill, J., Inder, T., Neil, J., Dierker, D., Harwell, J., and Van Essen, D. (2010). Similar patterns of cortical expansion during human development and evolution. Proc. Natl. Acad. Sci. U.S.A. 107, 13135-13140. doi: 10.1073/pnas.10012 29107

Kahn, I., Andrews-Hanna, J. R., Vincent, J. L., Snyder, A. Z., and Buckner, R. L. (2008). Distinct cortical anatomy linked to subregions of the medial temporal lobe revealed by intrinsic functional connectivity. J. Neurophysiol. 100, 129-139. doi: $10.1152 /$ jn. 00077.2008

Kahn, I., and Shohamy, D. (2013). Intrinsic connectivity between the hippocampus, nucleus accumbens, and ventral tegmental area in humans. Hippocampus 23, 187-192. doi: 10.1002/hipo.22077

Keller, C. J., Bickel, S., Honey, C. J., Groppe, D. M., Entz, L., Craddock, R. C., et al. (2013). Neurophysiological investigation of spontaneous correlated and anticorrelated fluctuations of the BOLD signal. J. Neurosci. 33, 6333-6342. doi: 10.1523/JNEUROSCI.4837-12.2013

Keller, J. B., Hedden, T., Thompson, T. W., Anteraper, S. A., Gabrieli, J. D., and Whitfield-Gabrieli, S. (2015). Resting-state anticorrelations between medial and lateral prefrontal cortex: association with working memory, aging, and individual differences. Cortex 64, 271-280. doi: 10.1016/j.cortex.2014.12.001

Kelly, A. M., Uddin, L. Q., Biswal, B. B., Castellanos, F. X., and Milham, M. P. (2008). Competition between functional brain networks mediates behavioral variability. Neuroimage 39, 527-537. doi: 10.1016/j.neuroimage.2007.08.008

Kennedy, K. M., Rodrigue, K. M., Bischof, G. N., Hebrank, A. C., Reuter-Lorenz, P. A., and Park, D. C. (2015). Age trajectories of functional activation under conditions of low and high processing demands: an adult lifespan fMRI study of the aging brain. Neuroimage 104, 21-34. doi: 10.1016/j.neuroimage.2014. 09.056

Koch, W., Teipel, S., Mueller, S., Buerger, K., Bokde, A. L., Hampel, H., et al. (2010). Effects of aging on default mode network activity in resting state fMRI: does the method of analysis matter? Neuroimage 51, 280-287. doi: 10.1016/j. neuroimage.2009.12.008

Kuhn, H. G., Dickinson-Anson, H., and Gage, F. H. (1996). Neurogenesis in the dentate gyrus of the adult rat: age-related decrease of neuronal progenitor proliferation. J. Neurosci. 15, 2027-2033.

Lebel, C., Gee, M., Camicioli, R., Wieler, M., Martin, W., and Beaulieu, C. (2012). Diffusion tensor imaging of white matter tract evolution over the lifespan. Neuroimage 60, 340-352. doi: 10.1016/j.neuroimage.2011.11.094

Levin, J. M., Frederick Bde, B., Ross, M. H., Fox, J. F., von Rosenberg, H. L., Kaufman, M. J., et al. (2001). Influence of baseline hematocrit and hemodilution on BOLD fMRI activation. Magn. Reson. Imaging 19, 1055-1062. doi: 10.1016/ S0730-725X(01)00460-X

Li, R., Chen, K., Fleisher, A. S., Reiman, E. M., Yao, L., and Wu, X. (2011). Large-scale directional connections among multi resting-state neural networks in human brain: a functional MRI and Bayesian network modeling study. Neuroimage 56, 1035-1042. doi: 10.1016/j.neuroimage.2011.03.010

Li, X., Pu, F., Fan, Y., Niu, H., Li, S., and Li, D. (2013). Age-related changes in brain structural covariance networks. Front. Hum. Neurosci. 7:98. doi: 10.3389/ fnhum.2013.00098

Li, Z., Moore, A. B., Tyner, C., and Hu, X. (2009). Asymmetric connectivity reduction and its relationship to "HAROLD" in aging brain. Brain Res. 1295, 149-158. doi: 10.1016/j.brainres.2009.08.004

Liang, Y., Sun, X., Xu, S., Liu, Y., Huang, R., Jia, J., et al. (2016). Preclinical cerebral network connectivity evidence of deficits in mild white matter lesions. Front. Aging Neurosci. 8:27. doi: 10.3389/fnagi.2016.00027

Lu, H., Xu, F., Rodrigue, K. M., Kennedy, K. M., Cheng, Y., Flicker, B., et al. (2011). Alterations in cerebral metabolic rate and blood supply across the adult lifespan. Cereb. Cortex 21, 1426-1434. doi: 10.1093/cercor/bhq224
Lu, T., Pan, Y., Kao, S. Y., Li, C., Kohane, I., Chan, J., et al. (2004). Gene regulation and DNA damage in the ageing human brain. Nature 429, 883-891. doi: 10. 1038/nature02661

Lynch, G., Rex, C. S., and Gall, C. M. (2006). Synaptic plasticity in early aging. Ageing Res. Rev. 5, 255-280. doi: 10.1016/j.arr.2006.03.008

Madden, D. J., Bennett, I. J., and Song, A. W. (2009). Cerebral white matter integrity and cognitive aging: contributions from diffusion tensor imaging. Neuropsychol. Rev. 19, 415-435. doi: 10.1007/s11065-009-9113-2

Mark, C. I., Mazerolle, E. L., and Chen, J. J. (2015). Metabolic and vascular origins of the BOLD effect: implications for imaging pathology and resting-state brain function. J. Magn. Reson. Imaging 42, 231-246. doi: 10.1002/jmri.24786

Marstaller, L., Williams, M., Rich, A., Savage, G., and Burianová, H. (2015). Aging and large-scale functional networks: white matter integrity, gray matter volume, and functional connectivity in the resting state. Neuroscience 290, 369-378. doi: 10.1016/j.neuroscience.2015.01.049

Mennes, M., Biswal, B. B., Castellanos, F. X., and Milham, M. P. (2013). Making data sharing work: the FCP/INDI experience. Neuroimage 82, 683-691. doi: 10.1016/j.neuroimage.2012.10.064

Menon, V. (2011). Large-scale brain networks and psychopathology: a unifying triple network model. Trends Cogn. Sci. 15, 483-506. doi: 10.1016/j.tics.2011. 08.003

Meunier, D., Achard, S., Morcom, A., and Bullmore, E. (2009). Age-related changes in modular organization of human brain functional networks. Neuroimage 44, 715-723. doi: 10.1016/j.neuroimage.2008.09.062

Montembeault, M., Joubert, S., Doyon, J., Carrier, J., Gagnon, J. F., Monchi, O., et al. (2012). The impact of aging on gray matter structural covariance networks. Neuroimage 63, 754-759. doi: 10.1016/j.neuroimage.2012.06.052

Moore, T. L., Killiany, R. J., Herndon, J. G., Rosene, D. L., and Moss, M. B. (2006). Executive system dysfunction occurs as early as middle-age in the rhesus monkey. Neurobiol. Aging 27, 1484-1493. doi: 10.1016/j.neurobiolaging.2005. 08.004

Mozley, P. D., Kim, H. J., Gur, R. C., Tatsch, K., Muenz, L. R., McElgin, W. T., et al. (1996). Iodine-123-IPT SPECT imaging of CNS dopamine transporters: nonlinear effects of normal aging on striatal uptake values. J. Nucl. Med. 37, 1965-1970.

Murphy, K., Birn, R. M., and Bandettini, P. A. (2013). Resting-state fMRI confounds and cleanup. Neuroimage 80, 349-359. doi: 10.1016/j.neuroimage. 2013.04.001

Murphy, K., Birn, R. M., Handwerker, D. A., Jones, T. B., and Bandettini, P. A. (2009). The impact of global signal regression on resting state correlations: are anti-correlated networks introduced? Neuroimage 44, 893-905. doi: 10.1016/j. neuroimage.2008.09.036

Murphy, K., and Fox, M. D. (2016). Towards a consensus regarding global signal regression for resting state functional connectivity MRI. Neuroimage doi: 10 . 1016/j.neuroimage.2016.11.052 [Epub ahead of print].,

Nichols, T. (2013). Notes on Creating a Standardized Version of DVARS [Internet]. Available at: http://www2.warwick.ac.uk/fac/sci/statistics/staff/academicresearch/nichols/scripts/fsl/standardizeddvars.pdf

Nyquist, P. A., Bilgel, M., Gottesman, R., Yanek, L. R., Moy, T. F., Becker, L. C., et al. (2015). Age differences in periventricular and deep white matter lesions. Neurobiol. Aging 36, 1653-1658. doi: 10.1016/j.neurobiolaging.2015.01.005

Onoda, K., Ishihara, M., and Yamaguchi, S. (2012). Decreased functional connectivity by aging is associated with cognitive decline. J. Cogn. Neurosci. 24, 2186-2198. doi: 10.1162/jocn_a_00269

Park, D. C., Lautenschlager, G., Hedden, T., Davidson, N. S., Smith, A. D., and Smith, P. K. (2002). Models of visuospatial and verbal memory across the adult life span. Psychol. Aging 17, 299-320. doi: 10.1037/0882-7974.17.2.299

Park, D. C., and Payer, D. (2006). "Working memory across the adult lifespan," in Lifespan Cognition: Mechanisms of Change, eds E. Bialystok and F. Craik (New York, NY: Oxford UP), 128-142.

Park, D. C., and Reuter-Lorenz, P. (2009). The adaptive brain: aging and neurocognitive scaffolding. Annu. Rev. Psychol. 60, 173-196. doi: 10.1146/ annurev.psych.59.103006.093656

Park, D. C., Smith, A. D., Lautenschlager, G., Earles, J. L., Frieske, D., Zwahr, M., et al. (1996). Mediators of long-term memory performance across the life span. Psychol. Aging 11, 621-637. doi: 10.1037/0882-7974.11.4.621

Park, H., Kennedy, K. M., Rodrigue, K. M., Hebrank, A., and Park, D. C. (2013). An fMRI study of episodic encoding across the lifespan: changes in subsequent 
memory effects are evident by middle-age. Neuropsychologia 51, 448-456. doi: 10.1016/j.neuropsychologia.2012.11.025

Patel, K. V. (2008). Variability and heritability of hemoglobin concentration: an opportunity to improve understanding of anemia in older adults. Haematologica 93, 1281-1283. doi: 10.3324/haematol. 13692

Patel, M. J., Boada, F. E., Price, J. C., Sheu, L. K., Tudorascu, D. L., Reynolds Iii, C. F., et al. (2012). Association of small vessel ischemic white matter changes with BOLD fMRI imaging in the elderly. Psychiatry Res. 204, 117-122. doi: 10.1016/j.pscychresns.2012.09.006

Peng, S. L., Dumas, J. A., Park, D. C., Liu, P., Filbey, F. M., McAdams, C. J., et al. (2014). Age-related increase of resting metabolic rate in the human brain. Neuroimage 98, 176-183. doi: 10.1016/j.neuroimage.2014. 04.078

Power, J. D., Barnes, K. A., Snyder, A. Z., Schlaggar, B. L., and Petersen, S. E. (2012). Spurious but systematic correlations in functional connectivity MRI networks arise from subject motion. Neuroimage 59, 2142-2154. doi: 10.1016/j. neuroimage.2011.10.018

Power, J. D., Mitra, A., Laumann, T. O., Snyder, A. Z., Schlaggar, B. L., and Petersen, S. E. (2014). Methods to detect, characterize, and remove motion artifact in resting state fMRI. Neuroimage 84, 320-341. doi: 10.1016/j.neuroimage.2013. 08.048

Power, J. D., Plitt, M., Laumann, T. O., and Martin, A. (2016). Sources and implications of whole-brain fMRI signals in humans. Neuroimage doi: 10.1016/ j.neuroimage.2016.09.038 [Epub ahead of print].

Raz, N. (2000). "Aging of the brain and its impact on cognitive performance: integration of structural and functional findings," in The Handbook of Aging and Cognition, eds F. Craik and T. A. Salthouse (Mahwah, NJ: Erlbaum), $1-90$.

Raz, N. (2001). “Ageing and the brain," in Encyclopedia of Life Sciences (London: Nature Publishing Group). Available at: https://www.researchgate.net/ publication/264402912_Ageing_and_the_Brain_2001_Encyclopedia_of_Life_ Sciences

Rex, C. S., Kramar, E. A., Colgin, L. L., Lin, B., Gall, C. M., and Lynch, G. (2005). Long-term potentiation is impaired in middle-aged rats: regional specificity and reversal by adenosine receptor antagonists. J. Neurosci. 25, 5956-5966. doi: 10.1523/JNEUROSCI.0880-05.2005

Rowe, J. B., Siebner, H., Filipovic, S. R., Cordivari, C., Gerschlager, W., Rothwell, J., et al. (2006). Aging is associated with contrasting changes in local and distant cortical connectivity in the human motor system. Neuroimage 32, 747-760. doi: 10.1016/j.neuroimage.2006.03.061

Salat, D. H., Tuch, D. S., Hevelone, N. D., Fischl, B., Corkin, S., Rosas, H. D., et al. (2005). Age-related changes in prefrontal white matter measured by diffusion tensor imaging. Ann. N. Y. Acad. Sci. 1064, 37-49. doi: 10.1196/annals. 1340.009

Salthouse, T. A. (2009). When does age-related cognitive decline begin? Neurobiol. Aging 30, 507-514. doi: 10.1016/j.neurobiolaging.2008.09.023

Scheinost, D., Finn, E. S., Tokoglu, F., Shen, X., Papademetris, X., Hampson, M., et al. (2015). Sex differences in normal age trajectories of functional brain networks. Hum. Brain Mapp. 36, 1524-1535. doi: 10.1002/hbm.22720

Seeley, W. W., Crawford, R. K., Zhou, J., Miller, B. L., and Greicius, M. D. (2009), Neurodegenerative diseases target large-scale human brain networks. Neuron 62, 42-52. doi: 10.1016/j.neuron.2009.03.024

Sheline, Y. I., Mintun, M. A., Moerlein, S. M., and Snyder, A. Z. (2002). Greater loss of 5-HT(2A) receptors in midlife than in late life. Am. J. Psychiatry 159, 430-435. doi: 10.1176/appi.ajp.159.3.430

Shoji, H., Takao, K., Hattori, S., and Miyakawa, T. (2016). Age-related changes in behavior in C57BL/6J mice from young adulthood to middle age. Mol. Brain 9:11. doi: 10.1186/s13041-016-0191-9

Singh-Manoux, A., Kivimaki, M., Glymour, M. M., Elbaz, A., Berr, C., Ebmeier, K. P., et al. (2012). Timing of onset of cognitive decline: results from Whitehall II prospective cohort study. BMJ 344:d7622. doi: 10.1136/bmj.d7622

Solesio-Jofre, E., Serbruyns, L., Woolley, D. G., Mantini, D., Beets, I. A., and Swinnen, S. P. (2014). Aging effects on the resting state motor network and interlimb coordination. Hum. Brain Mapp. 35, 3945-3961. doi: 10.1002/hbm. 22450

Song, J., Birn, R. M., Boly, M., Meier, T. B., Nair, V. A., Meyerand, M. E., et al. (2014). Age-related reorganizational changes in modularity and functional connectivity of human brain networks. Brain Connect. 4, 662-676. doi: 10.1089/ brain.2014.0286

Spreng, R. N., Stevens, W. D., Viviano, J. D., and Schacter, D. L. (2016). Attenuated anticorrelation between the default and dorsal attention networks with aging: evidence from task and rest. Neurobiol. Aging 45, 149-160. doi: 10.1016/j. neurobiolaging.2016.05.020

Stouffer, E. M., and Yoder, J. E. (2011). Middle-aged (12 month old) male rats show selective latent learning deficit. Neurobiol. Aging 32:2320. doi: 10.1016/ j.neurobiolaging.2010.04.021

Tau, G. Z., and Peterson, B. S. (2010). Normal development of brain circuits. Neuropsychopharmacology 35, 147-168. doi: 10.1038/npp.2009.115

Terribilli, D., Schaufelberger, M. S., Duran, F. L., Zanetti, M. V., Curiati, P. K., Menezes, P. R., et al. (2011). Age-related gray matter volume changes in the brain during non-elderly adulthood. Neurobiol. Aging 32, 354-368. doi: 10. 1016/j.neurobiolaging.2009.02.008

Thambisetty, M., Wan, J., Carass, A., An, Y., Prince, J. L., and Resnick, S. M. (2010). Longitudinal changes in cortical thickness associated with normal aging. Neuroimage 52, 1215-1223. doi: 10.1016/j.neuroimage. 2010. 04.258

Tomasi, D., and Volkow, N. D. (2012). Aging and functional brain networks. Mol. Psychiatry 17, 549-558. doi: 10.1038/mp.2011.81

Tsvetanov, K. A., Henson, R. N., Tyler, L. K., Razi, A., Geerligs, L., Ham, T. E., et al. (2016). Extrinsic and intrinsic brain network connectivity maintains cognition across the lifespan despite accelerated decay of regional brain activation. J. Neurosci. 36, 3115-3126. doi: 10.1523/JNEUROSCI.273315.2016

Turner, G. R., and Spreng, R. N. (2015). Prefrontal engagement and reduced default network suppression co-occur and are dynamically coupled in older adults: the default-executive coupling hypothesis of aging. J. Cogn. Neurosci. 27, 2462-2476. doi: 10.1162/jocn_a_00869

Van den Heuvel, M. P., and Hulshoff Pol, H. E. (2010). Exploring the brain network: a review on resting-state fMRI functional connectivity. Eur. Neuropsychopharmacol. 20, 519-534. doi: 10.1016/j.euroneuro.2010. 03.008

Van den Heuvel, M. P., Mandl, R. C., Kahn, R. S., and Hulshoff Pol, H. E. (2009). Functionally linked resting-state networks reflect the underlying structural connectivity architecture of the human brain. Hum. Brain Mapp. 30, 3127-3141. doi: 10.1002/hbm.20737

Van Dijk, K. R., Hedden, T., Venkataraman, A., Evans, K. C., Lazar, S. W., and Buckner, R. L. (2010). Intrinsic functional connectivity as a tool for human connectomics: theory, properties, and optimization. J. Neurophysiol. 103, $297-$ 321. doi: 10.1152/jn.00783.2009

Van Dijk, K. R., Sabuncu, M. R., and Buckner, R. L. (2012). The influence of head motion on intrinsic functional connectivity MRI. Neuroimage 59, 431-438. doi: 10.1016/j.neuroimage.2011.07.044

Van Helmond, Z., Miners, J. S., Kehoe, P. G., and Love, S. (2010). Higher soluble amyloid beta concentration in frontal cortex of young adults than in normal elderly or Alzheimer's disease. Brain Pathol. 20, 787-793. doi: 10.1111/j.17503639.2010.00374.x

Vidal-Piñeiro, D., Valls-Pedret, C., Fernández-Cabello, S., Arenaza-Urquijo, E. M., Sala-Llonch, R., Solana, E., et al. (2014). Default mode network connectivity correlates with age-associated structural and cognitive changes. Front. Aging Neurosci. 6:256. doi: 10.3389/fnagi.2014.00256

Villardita, C., Cultrera, S., Cupone, V., and Mejìa, R. (1985). Neuropsychological test performances and normal aging. Arch. Gerontol. Geriatr. 4, 311-319. doi: 10.1016/0167-4943(85)90038-X

Volkow, N. D., Gur, R. C., Wang, G. J., Fowler, J. S., Moberg, P. J., Ding, Y. S., et al. (1998). Association between decline in brain dopamine activity with age and cognitive and motor impairment in healthy individuals. Am. J. Psychiatry 155, 344-349.

Wu, T., Zang, Y., Wang, L., Long, X., Hallett, M., Chen, Y., et al. (2007). Aging influence on functional connectivity of the motor network in the resting state. Neurosci. Lett. 422, 164-168. doi: 10.1016/j.neulet.2007. 06.011

Yeo, B. T., Krienen, F. M., Sepulcre, J., Sabuncu, M. R., Lashkari, D., Hollinshead, M., et al. (2011). The organization of the human cerebral cortex estimated by intrinsic functional connectivity. J. Neurophysiol. 106, 1125-1165. doi: $10.1152 /$ jn. 00338.2011 
Ziegler, G., Dahnke, R., Jancke, L., Yotter, R. A., May, A., and Gaser, C. (2012). Brain structural trajectories over the adult lifespan. Hum. Brain Mapp. 33, 2377-2389. doi: $10.1002 / \mathrm{hbm} .21374$

Zuo, X. N., Kelly, C., Di Martino, A., Mennes, M., Margulies, D. S., Bangaru, S., et al. (2010). Growing together and growing apart: regional and sex differences in the lifespan developmental trajectories of functional homotopy. J. Neurosci. 30, 15034-15043. doi: 10.1523/JNEUROSCI.2612-10.2010

Conflict of Interest Statement: The authors declare that the research was conducted in the absence of any commercial or financial relationships that could be construed as a potential conflict of interest.
The reviewer NE and handling Editor declared their shared affiliation, and the handling Editor states that the process nevertheless met the standards of a fair and objective review.

Copyright $\odot 2017$ Siman-Tov, Bosak, Sprecher, Paz, Eran, Aharon-Peretz and Kahn. This is an open-access article distributed under the terms of the Creative Commons Attribution License (CC BY). The use, distribution or reproduction in other forums is permitted, provided the original author(s) or licensor are credited and that the original publication in this journal is cited, in accordance with accepted academic practice. No use, distribution or reproduction is permitted which does not comply with these terms. 